\title{
Organotin(IV) Esters of 4-Maleimido-benzoic Acid: Synthesis, Characterization and in vitro Anti-leishmanial Effects
}

\author{
M. I. Khan, *,a Musa Kaleem Baloch, ${ }^{b}$ Muhammad Ashfaq ${ }^{c}$ and Saima Gul ${ }^{a}$ \\ ${ }^{a}$ Department of Chemistry, Kohat University of Science \& Technology, Kohat-26000 (N-W.F.P.), Pakistan \\ ${ }^{b}$ Department of Chemistry, Gomal University, Dera Ismail Khan, Pakistan \\ ${ }^{c}$ Department of Chemistry, Islamia University, Bahawalpur, Pakistan
}

\begin{abstract}
Seis novos ésteres diorganoestanho(IV) com composição geral $\mathrm{R}_{2} \mathrm{SnL}_{2}$ (onde $\mathrm{R}$ : $\mathrm{Me}(\mathbf{1})$, $\operatorname{Et}(\mathbf{2}), \operatorname{Pr}(\mathbf{3}), \mathrm{Bu}(\mathbf{4}), \operatorname{Ph}(\mathbf{5}), \mathrm{Bz}(\mathbf{6})$ e L(7): ácido $p$ - $N$-maleimidobenzóico) foram sintetizados neste trabalho. Espectros de absorção no infravermelho e de Mössbauer de ${ }^{119 \mathrm{~m}} \mathrm{Sn}$ no estado sólido revelaram o comportamento bidentado de L em relação ao centro diorganoestanho(IV) nos complexos octaédricos distorcidos. Espectros de $\mathrm{RMN}$ de ${ }^{1} \mathrm{H},{ }^{13} \mathrm{C}$ e ${ }^{119} \mathrm{Sn}$, em $\mathrm{CDCl}_{3}$, indicaram hexacoordenação em 1-4, pentacoordenação de $\mathbf{5}$ em geometria trapezoidal distorcida, e ausência de hipercoordenação no arranjo tetraédrico em 6. Dados de análise elementar comprovaram a estequiometria dos compostos organoestanho(IV). Foram realizados testes in vitro contra cinco espécies de Leishmania: L. major, L. tropica, L. infantum, L. mex. mex. e L. donovani. Resultados promissores foram observados e, com base nos dados obtidos nesses ensaios, tentou-se estabelecer relações estrutura-atividade. O aumento no tamanho dos grupos $\mathrm{R}$ em $\left\{\mathrm{R}_{2} \mathrm{Sn}^{\mathrm{IV}}\right\}^{2+}$ aumentou a lipofilicidade dos complexos organoestanho(IV), acentuando assim a atividade antileishmania.
\end{abstract}

Six new diorganotin(IV) esters with the general composition $\mathrm{R}_{2} \mathrm{SnL}_{2}$ (where $\mathrm{R}$ : $\mathrm{Me}(\mathbf{1}), \operatorname{Et}(\mathbf{2})$, $\operatorname{Pr}(3), \operatorname{Bu}(4), \operatorname{Ph}(5), \mathrm{Bz}(\mathbf{6})$ and $\mathrm{L}(\mathbf{7}): p-N$-maleimido-benzoic acid) have been synthesized. Solid state FTIR and ${ }^{119 \mathrm{~m}} \mathrm{Sn}$ Mössbauer spectra revealed bidentate behavior of L towards the diorganotin(IV) centre in the distorted octahedral products. ${ }^{1} \mathrm{H},{ }^{13} \mathrm{C}$ and ${ }^{119} \mathrm{Sn}$ NMR spectra in $\mathrm{CDCl}_{3}$ indicated hexacoordination in $\mathbf{1 - 4}$, penta-coordination of $\mathbf{5}$ in skew-trapezoidal geometry, and absence of hypercoordination in tetrahedral 6. Elemental analyses data have been found to corroborate the stoichiometry of the title organotin(IV) compounds. In vitro anti-leishmanial screenings have been conducted on five leishmanial strains of L. major, L. tropica, L. infantum, L. mex. mex. and L. donovani. Promising results have been observed and, on the basis of the data obtained during these assays, a structure-activity relationship has been suggested. The increasing size of the R groups in the $\left\{\mathrm{R}_{2} \mathrm{Sn}^{\mathrm{IV}}\right\}^{2+}$ moieties increased the lipophilicity of organotin(IV) complexes, which thereby enhanced the anti-leishmanial activity.

Keywords: organotin(IV), anti-leishmanial, SAR

\begin{abstract}
Abbreviations
Me(methyl), Et(ethyl), Pr(n-propyl), Bu(n-butyl), $\mathrm{Ph}$ (phenyl), Bz(benzyl).
\end{abstract}

\section{Introduction}

Amino acids and their organic as well as organometallic derivatives present a wide range of noteworthy pharmacological applications. ${ }^{1}$ Transition metal complexes

*e-mail: chmikhan@hotmail.com of $\mathrm{N}$-protected amino acids are active against different types of microbes, but literature reveals that the coordinating ability of $N$-protected amino acids as ligands decrease the biological activity of their transition metal complexes up to a certain extent. ${ }^{2}$ Organotin(IV) compounds are well-known for their manifold implications, such as tumouricidal, bactericidal and fungicidal activities, and for their interesting structural features. ${ }^{3}$ Leishmaniasis is a parasitic disease in tropical countries and the number of leishmanial cases has increased alarmingly during the last decade. Triphenyltin(IV) complexes of salicylanilide thiosemicarbazone have been reported to be effective in vitro 
and in vivo as anti-leishmanial agents against L. donovani, and considered a good prospect as a therapeutic mediator for leishmaniasis. ${ }^{4}$ Keeping in view all these facts and our recent work, dealing with the synthesis of new cytotoxic organotin(IV) complexes utilizing biologically active molecules as ligands, ${ }^{4}$ in this communication we describe the synthesis and spectroscopic analyses of six new diorganotin(IV)-di-4-maleimido-benzoates, which have been screened in vitro for anti-leishmanial activity on five different leishmanial strains.

\section{Experimental}

\section{General}

Analytical Reagent (AR) grade chemicals used during this work were procured from Sigma or Fluka and used without purification. Dibenzyltin(IV) dichloride was prepared according to a reported procedure, and solvents were dried as reported. ${ }^{5}$

\section{Instrumentation}

Elemental analyses $(\mathrm{C}, \mathrm{H}, \mathrm{N})$ were performed on a Yanaco high-speed $\mathrm{CHN}$ analyzer with antipyrene as a reference, while tin content was estimated according to a reported procedure. ${ }^{5}$ Uncorrected melting points were taken on a Reichert Thermovar of F. G. Bode Co., Austria. The FTIR spectra of the $p$-N-maleimido-benzoic acid (L) and the complexes were measured on a Brüker FTIR TENSOR27spectrophotometer using OPUS software in the range of 5000-500 $\mathrm{cm}^{-1}$ (ZnSe). For Mössbauer measurements, the solid samples were maintained at liquid nitrogen temperature $(77.3 \mathrm{~K})$, and the equipment employed was a V.G. Micromass 7070 F Cryolid liquid nitrogen cryostat. The multichannel calibration was performed with an enriched iron foil using a ${ }^{57} \mathrm{Co}-\mathrm{Pd}$ source, while the zero point of the Doppler velocity scale was determined through the absorption spectra of $\mathrm{CaSnO}_{3}\left({ }^{119} \mathrm{Sn}=0.5 \mathrm{mg} \mathrm{cm}^{-2}\right)$. The resulting $5 \times 10^{5}$-count spectra were refined to obtain the isomer shift (IS), the nuclear quadrupole splitting (QS), $\rho$ (QS/IS) and the width at half-height of the resonant peaks, $\Gamma\left(\mathrm{mm} \mathrm{s}^{-1}\right) .{ }^{1} \mathrm{H}$ and ${ }^{13} \mathrm{C}$ NMR spectra in deuterated chloroform $\left(\mathrm{CDCl}_{3}\right)$ were recorded on a multinuclear Brüker Biospin AMX $300 \mathrm{MHz}$ FT NMR spectrometer operating at room temperature $\left(300 \mathrm{MHz}\right.$ for ${ }^{1} \mathrm{H}$ and $75 \mathrm{MHz}$ for ${ }^{13} \mathrm{C}$ ); the hydrogen and carbon chemical shifts were measured with respect to $\mathrm{SiMe}_{4} \cdot{ }^{119} \mathrm{Sn}$ NMR spectra in $\mathrm{CDCl}_{3}$ were recorded at $186.5 \mathrm{MHz}$ on a Brüker AMX 500 spectrophotometer using $5 \mathrm{~mm}$ o.d. tubes and are reported relative to external neat $\mathrm{SnMe}_{4}\left(\delta^{119} \mathrm{Sn}=0 \mathrm{ppm}\right)$.
Important parameters were acquisition time (AQ) of $3.6 \mathrm{~s}$, relaxation time (d1) $0.01 \mathrm{~s}$, sweep width (SW) $4545.45 \mathrm{~Hz}$ and number of data points (TD) 32768 .

\section{Synthesis of 4-maleimido-benzoic acid}

Maleic anhydride $\left(10 \mathrm{~g}, 0.1 \mathrm{~mol} \mathrm{~L}^{-1}\right)$ was dissolved in acetic acid $(150 \mathrm{~mL})$ and a cold solution of 4-aminobenzoic acid $\left(9.1 \mathrm{~g}, 0.1 \mathrm{~mol} \mathrm{~L}^{-1}\right)$ in acetic acid $(150 \mathrm{~mL})$ was added to it. This mixture was stirred at room temperature for $3 \mathrm{~h}$ resulting in a white precipitate, which was washed several times with cold water and recrystallized from water to give maleamic acid of analytical purity. Maleamic acid (5 g, $\left.0.02 \mathrm{~mol} \mathrm{~L}^{-1}\right)$ was then suspended in dry toluene $(350 \mathrm{~mL})$, and triethylamine $\left(7.5 \mathrm{~mL}, 0.05 \mathrm{~mol} \mathrm{~L}^{-1}\right)$ was added to this suspension. The mixture was refluxed with vigorous stirring for $1.5 \mathrm{~h}$ with the concomitant removal of water using a Dean-Stark separator. The solvent was removed on a rotary evaporator (Büchi) leaving a hygroscopic solid; $\mathrm{HCl}$ was added up to $\mathrm{pH} 2$ and the mixture was extracted with ethyl acetate and dried over anhydrous $\mathrm{MgSO}_{4}$. The ethyl acetate fraction was vacuum dried; the solid mass left was recrystallized from hexane. Figure 1 depicts a general chemical reaction scheme.

\section{Synthesis of organotin(IV) complexes}

A solution of the triethylammonium salt of 4-maleimidobenzoic acid $\left(0.5 \mathrm{~g}, 0.0015 \mathrm{~mol} \mathrm{~L}^{-1}\right)$ in dry toluene $(100 \mathrm{~mL})$ was prepared and an appropriate amount of diorganotin(IV) dichloride $\left(0.0008 \mathrm{~mol} \mathrm{~L}^{-1}\right)$ was added. This mixture was heated to reflux for 3 hours, resulting in the formation of triethylammonium hydrochloride, which was filtered off. The filtrate was evaporated on a rotary evaporator and the solid mass was triturated in $n$-hexane, dissolved in $\mathrm{C}_{6} \mathrm{H}_{6}$ and finally recrystallized from $\mathrm{CH}_{2} \mathrm{Cl}_{2}$.

\section{Spectral data for compounds (1-7)}

\section{Bis(4-maleimido-benzoato)dimethyltin(IV)(1)}

White solid, mp $158{ }^{\circ} \mathrm{C}$. Yield: $81 \%$. IR $v_{\max } / \mathrm{cm}^{-1}$ : $1631 v(\mathrm{COO})_{\mathrm{a}}, 1447 \mathrm{v}(\mathrm{COO})$, $\Delta v$ : 184, $411 \mathrm{v}(\mathrm{Sn}-\mathrm{O})$, $522 \mathrm{v}(\mathrm{Sn}-\mathrm{C})$ a, $517 \mathrm{v}(\mathrm{Sn}-\mathrm{C}){ }_{\mathrm{s}} \cdot{ }^{119 \mathrm{~m} S n}$ Mössbauer $\left(\mathrm{mm} \mathrm{s}^{-1}\right)$ : QS: 3.31, IS: $1.32, \Gamma_{1}: 0.98, \Gamma_{2}: 0.87, \rho: 2.50 .{ }^{1} \mathrm{H}$ NMR $\left(\mathrm{CDCl}_{3}\right) \delta 7.8(\mathrm{~d}, J 2.0 \mathrm{~Hz}, 1 \mathrm{H}, \mathrm{CH}), 7.7(\mathrm{~d}, J 7.1 \mathrm{~Hz}, 1 \mathrm{H}$, $\mathrm{CH}), 7.1$ (d, J 7.3 Hz, 1H, CH), $0.6\left(\mathrm{~s}, 3 \mathrm{H}, \mathrm{CH}_{3}\right) ;{ }^{13} \mathrm{C} \mathrm{NMR}$ $\left(75 \mathrm{MHz} \mathrm{CDCl}_{3}\right) \delta: 175.4(1 \mathrm{C}), 133.6(2 \mathrm{C}), 136.8(3 \mathrm{C})$; 129.0(4C), 136.4(5C), 170.0(6C), 135.4(7C), -0.7 (8C, $\left.{ }^{1} J\left({ }^{119} \mathrm{Sn}-{ }^{13} \mathrm{C}\right) 903 \mathrm{~Hz}\right)$. Anal. Calc. for $\mathrm{C}_{24} \mathrm{H}_{18} \mathrm{~N}_{2} \mathrm{O}_{8} \mathrm{Sn}: \mathrm{C}$, 49.52; H, 3.04; N, 4.82; Sn, 20.43. Found: C, 49.60; H, 3.12; N, 4.66; Sn, 20.29. 

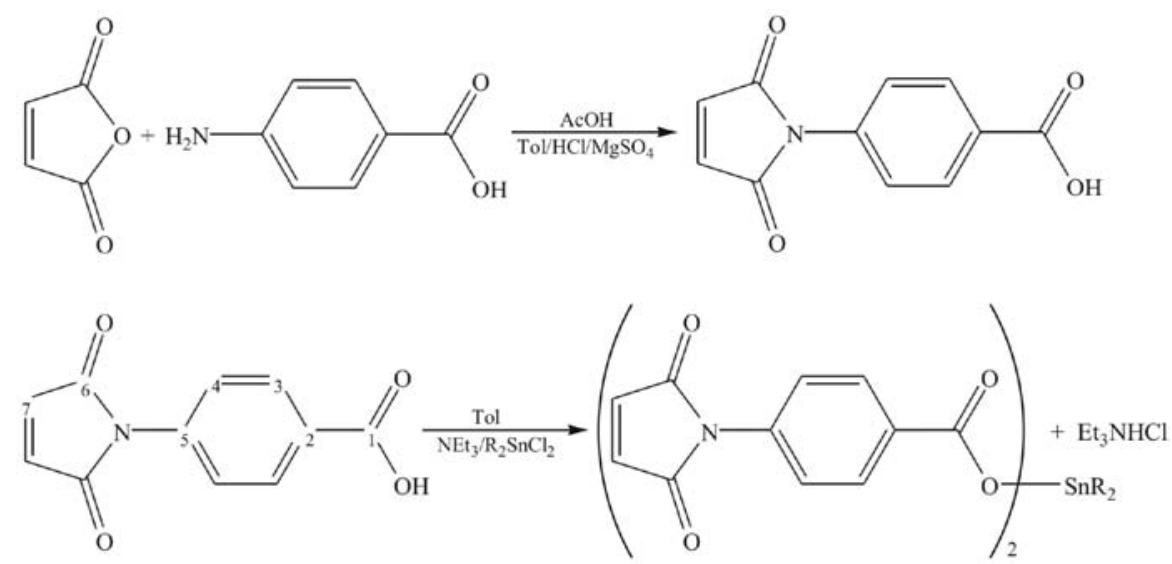

Figure 1. General reaction scheme for the synthesis of 4-maleimido-benzoic acid and its $\operatorname{tin}^{\mathrm{IV}}$ complexes.

Bis(4-maleimido-benzoato)diethyltin(IV)(2)

White solid, mp $74{ }^{\circ} \mathrm{C}$. Yield: $83 \%$. IR $v_{\max } / \mathrm{cm}^{-1}: 1655$ $v(\mathrm{COO}), 1474 v(\mathrm{COO}), \Delta v: 181,409 v(\mathrm{Sn}-\mathrm{O}), 537$ $v(\mathrm{Sn}-\mathrm{C}){ }_{\mathrm{a}}, 523 \mathrm{v}(\mathrm{Sn}-\mathrm{C})_{\mathrm{s}} \cdot{ }^{119 \mathrm{~m}} \mathrm{Sn}$ Mössbauer $\left(\mathrm{mm} \mathrm{s}^{-1}\right)$ : QS: 3.44, IS: $1.41, \Gamma_{1}: 0.96, \Gamma_{2}: 0.84, \rho: 2.43 .{ }^{1} \mathrm{H} \mathrm{NMR}\left(\mathrm{CDCl}_{3}\right)$ $\delta 7.8(\mathrm{~d}, J 2.0 \mathrm{~Hz}, 1 \mathrm{H}, \mathrm{CH}), 7.8(\mathrm{~d}, J 7.1 \mathrm{~Hz}, 1 \mathrm{H}, \mathrm{CH})$, 7.1 (d, J $7.3 \mathrm{~Hz}, 1 \mathrm{H}, \mathrm{CH}), 0.9\left(\mathrm{q},{ }^{2} J\left({ }^{119} \mathrm{Sn}-{ }^{1} \mathrm{H}\right) 100 \mathrm{~Hz}\right.$, $\left.2 \mathrm{H}, \mathrm{CH}_{2}\right), 0.8\left(\mathrm{t}, J 4.2 \mathrm{~Hz}, 3 \mathrm{H}, \mathrm{CH}_{3}\right) ;{ }^{13} \mathrm{C} \mathrm{NMR}(75 \mathrm{MHz}$, $\left.\mathrm{CDCl}_{3}\right) \delta: 168.5(1 \mathrm{C}), 130.1(2 \mathrm{C}), 137.0(3 \mathrm{C}) ; 131.3(4 \mathrm{C})$, 138.2(5C), 172.3(6C), 139.2(7C), 10.7(8C, ${ }^{1} J\left({ }^{119} \mathrm{Sn}-{ }^{13} \mathrm{C}\right)$ $918 \mathrm{~Hz}), 6.1\left(9 \mathrm{C},{ }^{2} \mathrm{~J}\left({ }^{119} \mathrm{Sn}-{ }^{13} \mathrm{C}\right) 187 \mathrm{~Hz}\right)$. Anal. Calc. for $\mathrm{C}_{26} \mathrm{H}_{22} \mathrm{~N}_{2} \mathrm{O}_{8} \mathrm{Sn}: \mathrm{C}, 51.46 ; \mathrm{H}, 3.64 ; \mathrm{N}, 4.60 ; \mathrm{Sn}, 19.19$. Found: C, 51.12; H, 4.37; N, 4.51; Sn, 19.03.

\section{Bis(4-maleimido-benzoato)dipropyltin(IV)(3)}

White solid, mp $94{ }^{\circ} \mathrm{C}$. Yield: $87 \%$. IR $v_{\max } / \mathrm{cm}^{-1}: 1651$ $v(\mathrm{COO})_{\mathrm{a}}, 1483 v(\mathrm{COO})_{\mathrm{s}}, \Delta v: 168,468 v(\mathrm{Sn}-\mathrm{O}), 529$ $v(\mathrm{Sn}-\mathrm{C}){ }_{\mathrm{a}}, 517 \mathrm{v}(\mathrm{Sn}-\mathrm{C})_{\mathrm{s}} \cdot{ }^{119 \mathrm{~m}} \mathrm{Sn}$ Mössbauer $\left(\mathrm{mm} \mathrm{s}^{-1}\right)$ : QS: 3.42, IS: $1.28, \Gamma_{1}: 1.00, \Gamma_{2}: 0.88, \rho: 2.67 .{ }^{1} \mathrm{H} \mathrm{NMR}\left(\mathrm{CDCl}_{3}\right)$ $\delta 7.8(\mathrm{~d}, J 2.0 \mathrm{~Hz}, 1 \mathrm{H}, \mathrm{CH}), 7.9(\mathrm{~d}, J 7.1 \mathrm{~Hz}, 1 \mathrm{H}, \mathrm{CH}), 7.0$ (d, J $7.3 \mathrm{~Hz}, 1 \mathrm{H}, \mathrm{CH}), 1.1\left(\mathrm{t},{ }^{2} J\left({ }^{119} \mathrm{Sn}-{ }^{-1} \mathrm{H}\right) 102 \mathrm{~Hz}, 2 \mathrm{H}\right.$, $\left.\mathrm{CH}_{2}\right), 1.6\left(\mathrm{~m}, 2 \mathrm{H}, \mathrm{CH}_{2}\right) 0.8\left(\mathrm{t}, J 4.2 \mathrm{~Hz}, 3 \mathrm{H}, \mathrm{CH}_{3}\right) ;{ }^{13} \mathrm{C} \mathrm{NMR}$ $\left(75 \mathrm{MHz}, \mathrm{CDCl}_{3}\right) \delta: 169.6(1 \mathrm{C}), 132.2(2 \mathrm{C}), 138.0(3 \mathrm{C})$; 131.0(4C), 136.9(5C), 171.1(6C), 135.1(7C), 34.2 (8C, $\left.{ }^{1} J\left({ }^{119} \mathrm{Sn}-{ }^{13} \mathrm{C}\right) 893 \mathrm{~Hz}\right), 20.3\left(9 \mathrm{C},{ }^{2} J\left({ }^{119} \mathrm{Sn}-{ }^{13} \mathrm{C}\right) 196 \mathrm{~Hz}\right)$. Anal. Calc. for $\mathrm{C}_{28} \mathrm{H}_{26} \mathrm{~N}_{2} \mathrm{O}_{8} \mathrm{Sn}$ : C, 52.78; H, 4.11; N, 4.40; Sn, 18.63. Found: C, 52.70; H, 4.02; N, 4.01; Sn, 18.45.

\section{Bis(4-maleimido-benzoato)dibutyltin(IV)(4)}

White solid, mp $138{ }^{\circ} \mathrm{C}$. Yield: $86 \%$. IR $v_{\max } / \mathrm{cm}^{-1}: 1596$ $v(\mathrm{COO})_{a}, 1431 v(\mathrm{COO}), \Delta v: 165,415 v(\mathrm{Sn}-\mathrm{O}), 547 v(\mathrm{Sn}-\mathrm{C})$, $531 \mathrm{v}(\mathrm{Sn}-\mathrm{C})_{\mathrm{s}^{\cdot}}{ }^{119 \mathrm{~m}} \mathrm{Sn}$ Mössbauer ( $\left.\mathrm{mm} \mathrm{s}^{-1}\right)$ : QS: 3.16, IS: 1.33, $\Gamma_{1}: 1.01, \Gamma_{2}: 0.94, \rho: 2.37 .{ }^{1} \mathrm{H}$ NMR $\left(\mathrm{CDCl}_{3}\right) \delta 7.8(\mathrm{~d}, J 2.0$ $\mathrm{Hz}, 1 \mathrm{H}, \mathrm{CH}), 7.7$ (d, J 7.1 Hz, 1H, CH), $7.0(\mathrm{~d}, J 7.3 \mathrm{~Hz}, 1 \mathrm{H}$, $\mathrm{CH}), 1.4\left(\mathrm{t},{ }^{2} J\left({ }^{119} \mathrm{Sn}-{ }^{1} \mathrm{H}\right) 99 \mathrm{~Hz}, 2 \mathrm{H}, \mathrm{CH}_{2}\right), 1.8\left(\mathrm{~m}, 2 \mathrm{H}, \mathrm{CH}_{2}\right)$, $1.3\left(\mathrm{~m}, 11 \mathrm{H}, \mathrm{CH}_{2}\right), 0.9$ (t, J $\left.4.2 \mathrm{~Hz}, 3 \mathrm{H}, \mathrm{CH}_{3}\right) ;{ }^{13} \mathrm{C} \mathrm{NMR}(75$
$\left.\mathrm{MHz}, \mathrm{CDCl}_{3}\right) \delta: 169.6(1 \mathrm{C}), 132.2(2 \mathrm{C}), 138.0(3 \mathrm{C}) ; 131.0(4 \mathrm{C})$, 136.9(5C), 171.9(6C), 134.1(7C), 27.1(14C, ${ }^{1} J\left({ }^{119} \mathrm{Sn}-{ }^{13} \mathrm{C}\right)$ $911 \mathrm{~Hz}), 27.3\left(15 \mathrm{C},{ }^{2} J\left({ }^{119} \mathrm{Sn}-{ }^{13} \mathrm{C}\right) 235 \mathrm{~Hz}\right)$. Anal. Calc. for $\mathrm{C}_{30} \mathrm{H}_{30} \mathrm{~N}_{2} \mathrm{O}_{8} \mathrm{Sn}: \mathrm{C}, 54.16 ; \mathrm{H}, 4.55 ; \mathrm{N}, 4.21 ; \mathrm{Sn}, 17.84$. Found: C, 54.01; H, 4.45; N, 4.13; Sn, 17.62 .

\section{Bis(4-maleimido-benzoato)diphenyltin(IV)(5)}

White solid, $\mathrm{mp} 83^{\circ} \mathrm{C}$. Yield: $71 \%$. IR $v_{\max } / \mathrm{cm}^{-1}: 1577$ $v(\mathrm{COO})_{\mathrm{a}}, 1425 v(\mathrm{COO})_{\mathrm{s}}, \Delta v: 152,400 v(\mathrm{Sn}-\mathrm{O}), 530$ $v(\mathrm{Sn}-\mathrm{C})_{\mathrm{a}}, 511 \mathrm{v}(\mathrm{Sn}-\mathrm{C})_{\mathrm{s}} \cdot{ }^{{ }^{1}}{ }^{119 \mathrm{~m}} \mathrm{Sn}$ Mössbauer $\left(\mathrm{mm} \mathrm{s}^{-1}\right)$ : QS: 3.38, IS: $1.04, \Gamma_{1}: 0.97, \Gamma_{2}: 0.96, \rho: 3.25 .{ }^{1} \mathrm{H} \mathrm{NMR}\left(\mathrm{CDCl}_{3}\right)$ $\delta 7.8(\mathrm{~d}, J 2.0 \mathrm{~Hz}, 1 \mathrm{H}, \mathrm{CH}), 7.6(\mathrm{~d}, J 7.1 \mathrm{~Hz}, 1 \mathrm{H}, \mathrm{CH}), 7.1$ $(\mathrm{d}, J 7.3 \mathrm{~Hz}, 1 \mathrm{H}, \mathrm{CH}), 7.8(\mathrm{~d}, J 7.7 \mathrm{~Hz}, 1 \mathrm{H}, \mathrm{CH}), 7.6(\mathrm{t}, J 7.7$ $\mathrm{Hz}, 1 \mathrm{H}, \mathrm{CH}), 1.3(\mathrm{t}, J 7.7 \mathrm{~Hz}, 1 \mathrm{H}, \mathrm{CH}) ;{ }^{13} \mathrm{C} \mathrm{NMR}(75 \mathrm{MHz}$, $\left.\mathrm{CDCl}_{3}\right) \delta: 170.0(1 \mathrm{C}), 129.9(2 \mathrm{C}), 136.8(3 \mathrm{C}) ; 132.3(4 \mathrm{C})$, 138.7(5C), 170.0(6C), 139.0(7C), 129.1(8C, ${ }^{1} J\left({ }^{119} \mathrm{Sn}-{ }^{13} \mathrm{C}\right)$ $326 \mathrm{~Hz}), 122.1\left(9 \mathrm{C},{ }^{2} J\left({ }^{119} \mathrm{Sn}-{ }^{13} \mathrm{C}\right) 154 \mathrm{~Hz}\right), 135.4(10 \mathrm{C}$, $\left.{ }^{3} J\left({ }^{119} \mathrm{Sn}-{ }^{13} \mathrm{C}\right) 198 \mathrm{~Hz}\right), 130.1\left(11 \mathrm{C},{ }^{4} J\left({ }^{119} \mathrm{Sn}-{ }^{13} \mathrm{C}\right) 123 \mathrm{~Hz}\right)$. Anal. Calc. for $\mathrm{C}_{34} \mathrm{H}_{22} \mathrm{~N}_{2} \mathrm{O}_{8} \mathrm{Sn}$ : C, 57.90; H, 3.14; N, 3.97; Sn, 16.83. Found: C, 57.79; H, 3.09; N, 3.74; Sn, 16.59.

\section{Bis(4-maleimido-benzoato)dibenzyltin(IV)(6)}

White solid, $\mathrm{mp} 142{ }^{\circ} \mathrm{C}$. Yield: $89 \%$. IR $v_{\max } / \mathrm{cm}^{-1}$ : $1593 v(\mathrm{COO})_{\mathrm{a}}, 1424 v(\mathrm{COO}), \Delta v: 169,416 v(\mathrm{Sn}-\mathrm{O}), 564$ $v(\mathrm{Sn}-\mathrm{C})_{\mathrm{a}}, 534 \mathrm{v}(\mathrm{Sn}-\mathrm{C})_{\mathrm{s}} \cdot{ }^{119 \mathrm{~m}} \mathrm{Sn}$ Mössbauer $\left(\mathrm{mm} \mathrm{s}^{-1}\right)$ : QS: 3.29, IS: $1.54, \Gamma_{1}: 0.98, \Gamma_{2}: 0.92, \rho: 2.13 .{ }^{1} \mathrm{H} \mathrm{NMR}\left(\mathrm{CDCl}_{3}\right)$ $\delta 7.7(\mathrm{~d}, J 2.0 \mathrm{~Hz}, 1 \mathrm{H}, \mathrm{CH}), 7.7(\mathrm{~d}, J 7.1 \mathrm{~Hz}, 1 \mathrm{H}, \mathrm{CH}), 7.1$ (d, J 7.3 Hz, 1H, CH), 3.0 (s, 2H, $\left.\mathrm{CH}_{2}\right), 7.7(\mathrm{~m}, 1 \mathrm{H}, \mathrm{CH})$, $7.3(\mathrm{~d}, J 5.2 \mathrm{~Hz}, 1 \mathrm{H}, \mathrm{CH}), 7.4(\mathrm{~m}, 1 \mathrm{H}, \mathrm{CH}) ;{ }^{13} \mathrm{C} \mathrm{NMR}$ $\left(75 \mathrm{MHz}, \mathrm{CDCl}_{3}\right.$ ) $\delta: 168.3(1 \mathrm{C}), 130.5(2 \mathrm{C}), 137.4(3 \mathrm{C})$; 134.3(4C), 137.2(5C), 169.8(6C), 136.7(7C), 20.6(8C, $\left.{ }^{1} J\left({ }^{19} \mathrm{Sn}-{ }^{13} \mathrm{C}\right) 401 \mathrm{~Hz}\right), 142.1\left(9 \mathrm{C},{ }^{2} J\left({ }^{119} \mathrm{Sn}-{ }^{13} \mathrm{C}\right) 174 \mathrm{~Hz}\right)$, $127.5\left(10 \mathrm{C},{ }^{3} J\left({ }^{119} \mathrm{Sn}-{ }^{13} \mathrm{C}\right) 223 \mathrm{~Hz}\right), 129.4(11 \mathrm{C}), 125.3(12 \mathrm{C}$, $\left.{ }^{4} J\left({ }^{119} \mathrm{Sn}-{ }^{13} \mathrm{C}\right) 231 \mathrm{~Hz}\right)$. Anal. Calc. for $\mathrm{C}_{36} \mathrm{H}_{26} \mathrm{~N}_{2} \mathrm{O}_{8} \mathrm{Sn}: \mathrm{C}$, 58.96; H, 3.57; N, 3.82; Sn, 16.19. Found: C, 58.74; H, 3.57 ; N, 3.76; Sn, 15.97 . 
4-maleimido-benzoic acid (7)

White solid, mp $109^{\circ} \mathrm{C}$. Yield: $89 \%$. IR $v_{\max } / \mathrm{cm}^{-1}: 1680$ $v(\mathrm{COO})_{\mathrm{a}}, 1405 \mathrm{v}(\mathrm{COO}), \Delta v: 275,3375-2870 v(\mathrm{O}-\mathrm{H})$. ${ }^{1} \mathrm{H}$ NMR $\left(\mathrm{CDCl}_{3}\right) \delta 7.9(\mathrm{~d}, J 2.0 \mathrm{~Hz}, 1 \mathrm{H}, \mathrm{CH}), 7.6(\mathrm{~d}$, J $7.1 \mathrm{~Hz}, 1 \mathrm{H}, \mathrm{CH}), 7.2(\mathrm{t}, J 7.3 \mathrm{~Hz}, 1 \mathrm{H}, \mathrm{CH}),{ }^{13} \mathrm{C} \mathrm{NMR}$ $\left(75 \mathrm{MHz}, \mathrm{CDCl}_{3}\right) \delta: 166.8(1 \mathrm{C}), 131.6(2 \mathrm{C}), 135.7(3 \mathrm{C})$; 129.6(4C), 140.4(5C), 165.9(6C), 135.1(7C). Anal. Calc. for $\mathrm{C}_{11} \mathrm{H}_{7} \mathrm{NO}_{4}$ : C, 60.83; H, 3.25; N, 6.45. Found: C, 59.68; $\mathrm{H}, 3.15 ; \mathrm{N}, 6.26$.

\section{In vitro anti-leishmanial activity}

All promastigote cultures of both the reference and local Pakistani leishmanial strains were maintained in blood agar based bi-phasic Evans modified Tobies medium supplemented with RPMI-1640 with $25 \mathrm{mmol} \mathrm{L}^{-1} \mathrm{TES}$ at $25^{\circ} \mathrm{C}$. Leishmanial strains in promastigote stage that were used include L. major (JISH118), L. major (MHOM/PK/88/ DESTO), L. tropica (K27), L. infantum (LEM3437), L. mex. mex. (LV4) and L. donovani (H43).

\section{Viability test}

Parasites in the promastigote stage were transferred from Evans modified to RPMI-1640 supplemented with 5\% fetal bovine serum (FBS) and 1\% sterile human urine, buffered with $25 \mathrm{mmol} \mathrm{L}^{-1} \mathrm{TES}, \mathrm{pH} 7.2$ (complete medium). They were grown in bulk at $25^{\circ} \mathrm{C}$ and then centrifuged at 2500 rev. per min for 10 min; early log phase promastigotes were collected. The parasites were washed twice with RPMI (without FBS or urine) and resuspended in the complete medium to achieve a final concentration of $10^{6}$ parasites per $\mathrm{mL}$. In order to get the $50 \%$ mortality concentration $\left(\mathrm{IC}_{50}\right)$, serial dilutions of the test compounds were performed in 96-well microtiter plate. Subsequently, $10^{5}$ promastigotes in $100 \mu \mathrm{L}$ of culture medium were added to each well and the plate was incubated at $25^{\circ} \mathrm{C}$ for $72 \mathrm{~h}$. Negative controls (culture without test compounds) were on the same plate. At the end of the incubation time, the plate was shaken mechanically over a plane shaker and parasites were counted with the help of a hemocytometer. Dose-dependent viability curves were obtained.

\section{Results and Discussion}

The ligand 4-maleimido-benzoic acid and its diorganotin(IV) complexes were synthesized by a general procedure as shown in Figure 1. Analytical data for the complexes confirmed the 1:2 metal-ligand stoichiometry. All compounds were quite stable with good yield (70-92\%) and were soluble in organic solvents. Elemental analysis data were found to be in good agreement with calculated contents.

\section{Molecular structure}

Solid-state FT IR spectra were recorded in the spectral range of 4000-400 $\mathrm{cm}^{-1}$ and important $v_{a}(\mathrm{COO}), v_{s}(\mathrm{COO})$, $v_{a}(\mathrm{Sn}-\mathrm{C}), v_{s}(\mathrm{Sn}-\mathrm{C}), v_{a}(\mathrm{Sn}-\mathrm{O})$ vibrational frequencies were observed in this region. The complexation of $\left\{\mathrm{R}_{2} \mathrm{Sn}^{\mathrm{IV}}\right\}^{2+}$ moieties with 4-maleimido-benzoic acid was confirmed by the absence of the broad band (1-6) of $v(\mathrm{OH})$ due to $\mathrm{COOH}$ group (7) in the spectral range of $3000-2600 \mathrm{~cm}^{-1} .6$ The imide $\mathrm{v}(\mathrm{N}-\mathrm{C}=\mathrm{O})$ band in the range of $1700-1710 \mathrm{~cm}^{-1}$ remained unchanged, which ruled out the interaction of $\mathrm{Sn}^{\mathrm{IV}}$ with imide CO. ${ }^{6}$

It is reported in the literature that the difference $(\Delta v)$ between $v_{a}(\mathrm{COO})$ and $v_{s}(\mathrm{COO})$ is important in predicting the coordinating ability of the ligand; in complexes 1-5, $\Delta \mathrm{v}$ was less than $200 \mathrm{~cm}^{-1}$, which indicated the bidentate nature of 4-maleimido-benzoic acid (Figure 2a). ${ }^{7}$ In the spectrum of 5 , a characteristic sharp peak at $450 \mathrm{~cm}^{-1}$ confirmed the $\mathrm{Sn}-\mathrm{Ph}$ bond. ${ }^{7}$ In addition, bands of medium intensity observed in the spectral ranges of $570-545 \mathrm{~cm}^{-1}$ and $490-430 \mathrm{~cm}^{-1}$ confirmed the presence of $\mathrm{Sn}-\mathrm{C}\left\{\mathrm{v}_{a}(\mathrm{Sn}-\mathrm{C})\right.$, $\left.v_{s}(\mathrm{Sn}-\mathrm{C})\right\}$ and $\mathrm{Sn}-\mathrm{O}$ bonds respectively. ${ }^{4}$

${ }^{119 m} \mathrm{Sn}$ Mössbauer spectroscopy provides useful information on the geometry around the tin atom in the solid state. ${ }^{8}$ In particular, quadropole splitting (QS) values often allow the discrimination between tetra- and hypercoordination of $\mathrm{Sn}^{\mathrm{IV}}$ centres; each of these being identified by characteristic value ranges (tetrahedral: $2.01-2.5 \mathrm{~mm} \mathrm{~s}^{-1}$, trigonalbipyramidal: 3.0-4.0 $\mathrm{mm} \mathrm{s}^{-1}$, cis-octahedral: $1.7-2.2 \mathrm{~mm} \mathrm{~s}^{-1}$, trans-octahedral: 3.0-4.5 $\left.\mathrm{mm} \mathrm{s}^{-1}\right){ }^{7}$ For diorganotin(IV) dicarboxylates, the $\rho$ values (QS/IS) play an important role in the prediction of the geometry around the tin centre; it is reported in the literature that if the $\rho$ value is greater than 2.1 , the dirganotin(IV) dicarboxylates possess a trans-octahedral geometry around the tin atom. Hence, in this work, $\rho$ values strongly suggest a trans-octahedral geometry for 1-4 (Figure 2a). ${ }^{9}$

The $\mathrm{CDCl}_{3}$ NMR spectra of 1-6 exhibited the expected resonances arising from the organotin(IV) moieties and hydrogens of 4-maleimido-benzoic acid. ${ }^{10}$ The coupling constants ${ }^{1} J\left[{ }^{119} \mathrm{Sn}-{ }^{13} \mathrm{C}\right]$ and ${ }^{2} J\left[{ }^{119} \mathrm{Sn}-{ }^{1} \mathrm{H}\right]$ yield important structural information; the magnitude of these coupling constants was consistent with a six-coordinate tin centre in an octahedral arrangement, indicating a 1:2 metalto-ligand stoichiometry. ${ }^{11}$ Howard's equations (1 and 2) were successfully applied for the estimation of $\mathrm{C}-\mathrm{Sn}-\mathrm{C}$ angle; equation (1) yielded $182^{\circ}, 186^{\circ}$ and $175^{\circ}$, indicating octahedron for compounds $\mathbf{2 - 4} .^{12}$

$$
\begin{aligned}
& \theta(\mathrm{C}-\mathrm{Sn}-\mathrm{C})=2.28{ }^{2} J\left[{ }^{119} \mathrm{Sn}-{ }^{1} \mathrm{H}\right]-46.4 \\
& \theta(\mathrm{C}-\mathrm{Sn}-\mathrm{C})=0.178{ }^{1} J\left[{ }^{119} \mathrm{Sn}-{ }^{13} \mathrm{C}\right]+14.7
\end{aligned}
$$




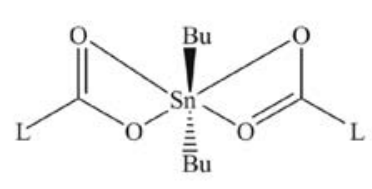

(a)

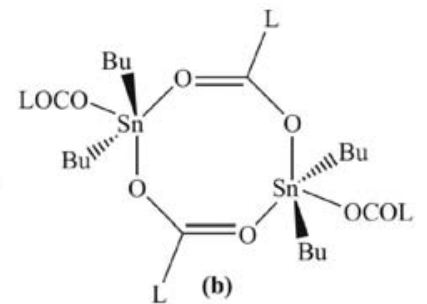

(b)<smiles>[3H]C(=O)O[13C@]([13CH3])([18OH])OC(C)=O</smiles>

(c)

R: ${ }^{8} \mathrm{CH}_{3}(1){ }^{8} \mathrm{CH}_{2} \cdot{ }^{9} \mathrm{CH}_{3}(2){ }^{8} \mathrm{CH}_{2}{ }^{-9} \mathrm{CH}_{2}-{ }^{10} \mathrm{CH}_{3}(3){ }^{8} \mathrm{CH}_{2}-{ }^{-} \mathrm{CH}_{2}{ }^{10} \mathrm{CH}_{2}-{ }^{11} \mathrm{CH}_{3}(4)$

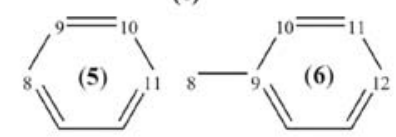

Figure 2. Geometrical trends in compounds 1-6 (a) trans-octahedral; (b) skew-trapezoidal; (c) tetrahedral; and numbering scheme for ${ }^{1} \mathrm{H}$ and ${ }^{13} \mathrm{C} \mathrm{NMR}$.

Equation (2), in its turn, employing ${ }^{1} J\left[{ }^{19} \mathrm{Sn}-{ }^{13} \mathrm{C}\right]$ values, provided $\mathrm{C}-\mathrm{Sn}-\mathrm{C}$ angles of $176^{\circ}, 179^{\circ}, 174^{\circ}$ and $177^{\circ}$ respectively for $\mathbf{1 - 4}$, confirming the trans-octahedral arrangement.

${ }^{119} \mathrm{Sn}$ NMR spectroscopy plays a significant role in the determination of geometry around tin atoms. ${ }^{13}{ }^{119}$ Sn NMR chemical shifts of 1-4 (-220.3, -208.5, -213.6 and -221.8 ppm) were comparable with earlier reports describing octahedral geometry. ${ }^{14}$ On the other hand, $\mathbf{5}$ showed a broad singlet at $-114.3 \mathrm{ppm}$, indicating the existence of an equilibrium between penta and hexa-coordination states describing a skew trapezoidal geometry (Figure 2b), while $\mathbf{6}$ displayed a resonance peak at $-44.6 \mathrm{ppm}$ characteristic of a tetrahedral $\mathrm{Sn}^{\mathrm{IV}}$ centre; in this case, coordination may be lost due to the size of the benzyl group. ${ }^{15}$ The coupling constant $\left[{ }^{1} J\left({ }^{119} \mathrm{Sn}-{ }^{13} \mathrm{C}\right)\right]$ furnished a typical trend, i.e., ${ }^{1} J>{ }^{2} J\left\langle{ }^{3} J\right.$, which confirmed the tetrahedral geometry of 6 (Figure 2c). ${ }^{15}$ These results were comparable to the solid state geometrical behavior of the complexes, confirming the 1:2 metal-to-ligand stoichiometry in the solid as well as in $\mathrm{CDCl}_{3}$ for all complexes.

\section{Bioactivity}

Table 1 contains the in vitro anti-leishmanial activity data of 1-7 and two reference drugs used clinically (Amphotericin B and Pentamidine). These displayed an anti-leishmanial activity trend as $\mathbf{7}<\mathbf{1}<\mathbf{2}<\mathbf{3}<\mathbf{4}<\mathbf{5}<$ 6 > > A and B (A: Amphotericin B, B: Pentamidine). The results obtained have been depicted in Figure 3, which suggests that the nature and size of the $\mathrm{R}$ group attached to $\mathrm{Sn}^{\mathrm{IV}}$ affect the in vitro anti-leishmnial activity. For highlighting this statement, the mean values of the average $\mathrm{IC}_{50}$ for compounds 1-7 against each leishmanial strain have been plotted versus the percent $\mathrm{CH}$ of $\mathrm{R}$ groups attached to $\mathrm{Sn}^{\mathrm{IV}}$ in Figure 4. The percent $\mathrm{CH}$ for compounds 1-7 were calculated as:

Percent $\mathrm{CH}(\mathrm{R})=\frac{\left[\mathrm{C}_{\mathrm{n}} \times(12.011)+\mathrm{H}_{\mathrm{n}} \times(1.0079)\right]}{\text { Molecular Mass of the Complex }} \times 100$

where $\mathrm{n}$ is the number of carbon or hydrogen atoms in $\mathrm{R}$ groups.

Figure 4 shows that the lethality increases almost linearly (and therefore $\mathrm{IC}_{50}$ decreases) with the increase in percent $\mathrm{CH}$ of the $\mathrm{R}$ groups. Some deviations in the case of smaller alkyl groups have been observed, which may be attributed to variation in the conformational behavior and distribution of complexes between phases. Literature reveals that organotin(IV) compounds formed with ligands containing carboxylic groups (-COO-Sn bonds) have proved to be more biologically active; the use of 4-maleimido-benzoic acid as ligand increases the hydrolysability of the organotin(IV) precursors due to formation of the Sn-O bonds. ${ }^{16}$ This property of the ligand permits attack of the hydrolysed $\left\{\mathrm{R}_{2} \mathrm{Sn}^{\mathrm{IV}}\right\}^{2+}$ moieties on the target cells, thereby enhancing the anti-leishmanial activity. ${ }^{16}$

On the other hand, the function of the R group is to determine the extent of activity; in this work, the trend was observed that the increase in the size of $\mathrm{R}$ groups made

Table 1. In vitro anti-leishmanial effect of 1-7 and standard drugs (A: Amphotericin B and B: Pentamidine, $\mathrm{IC}_{50}$ in $\mu \mathrm{g} \mathrm{mL}^{-1}$ )

\begin{tabular}{|c|c|c|c|c|c|c|c|c|c|}
\hline Leishm. strain & (1) & (2) & (3) & $\begin{array}{l}\text { mpol } \\
\text { (4) }\end{array}$ & (5) & (6) & (7) & $\mathbf{A}$ & B \\
\hline L. major & 144 & 151 & 127 & 101 & 89 & 66 & 202 & 128 & 203 \\
\hline$L$ major (Pak.) & 225 & 177 & 142 & 123 & 100 & 95 & 184 & 101 & 147 \\
\hline L. tropica & 88 & 76 & 44 & 23 & 11 & 03 & 107 & 175 & 203 \\
\hline L. mex. mex. & 112 & 110 & 87 & 69 & 65 & 49 & 142 & 126 & 167 \\
\hline L. donovani & 100 & 58 & 54 & 26 & 14 & 08 & 116 & 131 & 108 \\
\hline
\end{tabular}




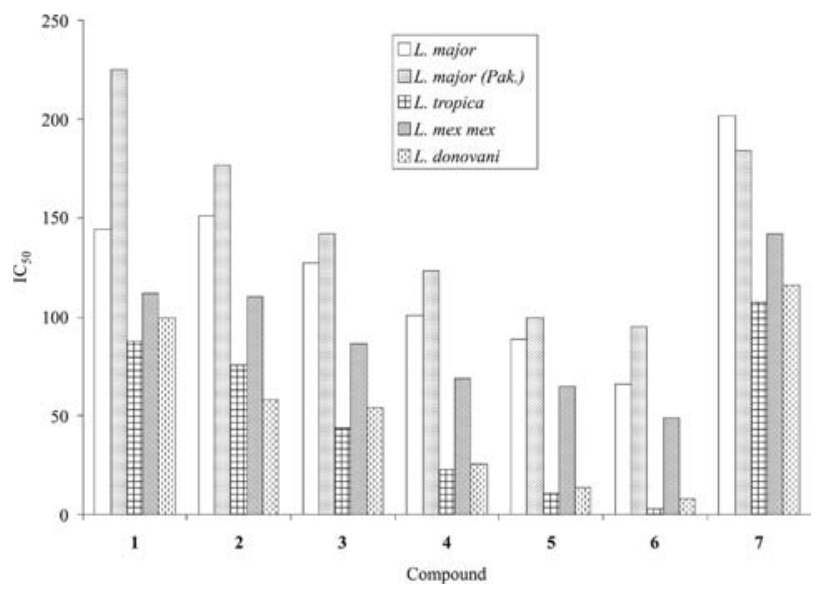

Figure 3. Effect of R groups on anti-leishmanial activity $\left(\mathrm{IC}_{50}\right)$ of 1-6.

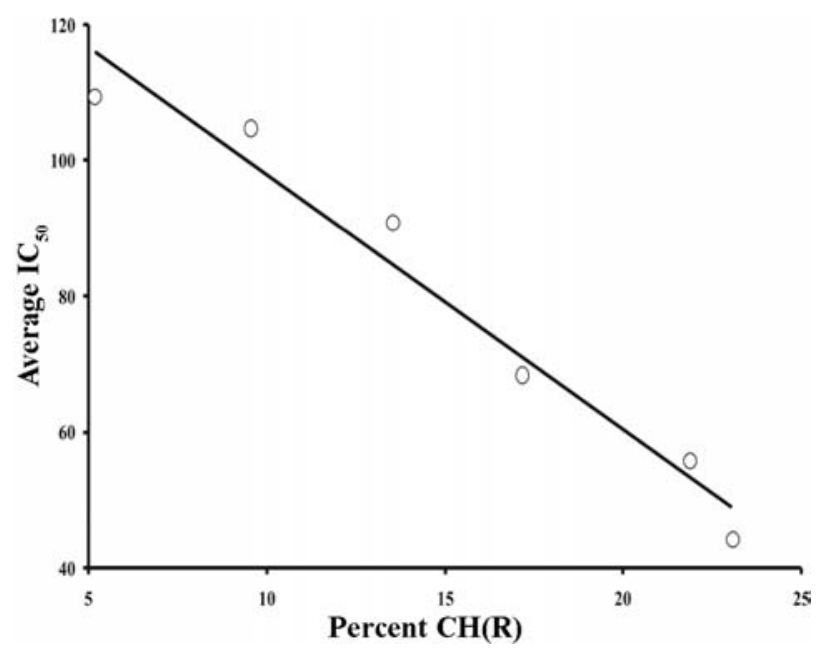

Figure 4. Effect of bulkier $\mathrm{R}$ groups (percent $\mathrm{CH}$ ) on anti-leishmanial activity $\left(\mathrm{IC}_{50}\right)$.

the $\left\{\mathrm{R}_{2} \mathrm{Sn}^{\mathrm{IV}}\right\}^{2+}$ moieties more lipophilic, resulting in the decrease of $\mathrm{IC}_{50}$ when compared with reference drugs (A and $\mathbf{B}$ ) and starting organotin(IV) reagents. Conclusively, we can say that the bulkiness of the attached $\mathrm{R}$ group/ percent $\mathrm{CH}$ values and polar character of carboxylic group of 4-maleimido-benzoic acid are interlinked with each other, and enhances the polarity $\mathrm{C}-\mathrm{Sn}$ and $\mathrm{O}-\mathrm{Sn}$ bonds in 1-6. A study is being carried out for the in vivo interactions/ mechanism of action of these complexes.

\section{Acknowledgment}

This work was carried out with the financial support of Gomal University, D. I. Khan, Pakistan, (Research Project No. $717-29 / \mathrm{DF} / \mathrm{GU})$.

\section{Supplementary Information}

Supplementary data are available free of charge at http:// jbcs.sbq.org.br, as PDF file.

\section{References}

1. Griffiths, D. G.; Partice, M. G.; Sharp, R. N.; Beechey, R. R.; FEBS Lett. 1981, 134, 261; Morder, L.; Biol. Chem. HoopeSeyler 1987, 368, 855; Rich, D. H.; Gasellchen, P. G.; Tong, A.; Cheung, A.; Buckner, C. K.; J. Med. Chem. 1975, 18, 1004; Codecik, E.; Reddi, K. K.; Nature 1951, 168, 475; Keller, O.; Rudinger, J.; Helv. Chim. Acta 1975, 58, 531; Nath, M.; Pokharia, S.; Yadav, R.; Coord. Chem. Rev. 2001, 215, 99; Gielen, M.; Appl. Organomet. Chem. 2002, 16, 481; Chandrasekhar, V.; Najendran, S.; Baskar, V.; Coord. Chem. Rev. 2002, 235, 1.

2. West, D. X.; Billeh, I. S.; Jasinski, J. P.; Butcher, R. J.; Transition Met. Chem. 1998, 23, 209; Costa, R. E. F.; Perez-Rebolledo, A.; Matensio, T.; Calado, H. D. R.; Adisson, J. D.; Cortés, M. E.; J. Coord. Chem. 2005, 1307, 58, 1307.

3. Gielen, M.; J. Braz. Chem. Soc. 2003, 14, 870.

4. Raychaudhury, B.; Banerjee, S.; Gupta, S.; Singh R. V.; Datta, S. C.; Acta Trop. 2005, 95, 1; Khan, M. I.; Baloch, M. K.; Ashfaq, M.; J. Enz. Inhib. Med. Chem. 2007, 22, 343; Khan, M. I.; Baloch, M. K.; Ashfaq, M.; Rehmat M.; Main Group Met. Chem. 2006, 29, 201; Khan, M. I.; Baloch, M. K.; Ashfaq, M.; Malik, A.; Mehsud Saifullah; Main Group Met. Chem. 2006, 29, 343; Ashfaq, M.; Khan, M. I.; Baloch, M. K.; Malik, A.; J. Organomet. Chem. 2004, 689, 238.

5. Sisido, K.; Takeda, Y.; Kinugawa, Z.; J. Am. Chem. Soc. 1961, 83, 538; Perrin, D. D.; Armarego, W. L. F.; Purification of Laboratory Chemicals, Butterworth Heinemann: Oxford, 1988; Mendham, J.; Denney, R. C.; Barnes, J. D.; Thomas, M.; Vogel's Text Book of Quantitative Chemical Analysis, $6^{\text {th }}$ ed., Pearson Education Pte. Ltd, Singapore, 2003.

6. Rehman, W.; Baloch, M. K.; Badshah, A.; J. Braz. Chem. Soc. 2005, 16, 827; Ewing, G. W.; Instrumental Methods of Chemical Analysis, McGraw-Hill: New York, 1985; Szymanski, H. A.; Erickson R .E.; Infrared Band Handbook, IFI/Plenum: New York, 1970.

7. Xueqing, D.; Qinglan, F.; Xiaoniu, F.; Heteroat. Chem. 2002, 13, 592; Xie, Q. L.; Yang, Z. Q.; Zhang, Z. X.; Zhang, D. K.; Appl. Organomet. Chem. 1992, 6, 193; Whiffen, D. H.; J. Chem. Soc. 1956, 1350

8. Parish, R. V.; Long, G. J.; Mössbauer Spectroscopy Applied to Inorganic Chemistry, Plenum Press: New York, 1984; Zhao, A.; Carraher, C.E.; Barone, G.; Pellerito, C.; Scopelliti, M.; Pellerito, L.; Polym. Mater. Sci. Eng. 2005, 93, 414; Barbieri, R.; Huber, F.; Pellerito, L.; Ruisi, G.; Silvestri, A.; Smith, P. J.; Chemistry of Tin: ${ }^{119}$ Sn Mössbauer Studies on Tin Compounds, Blackie: London, 1988.

9. Gielen. M.; Khloufi, A. El; Biesemans, M.; Kayser, F.; Willem, F.; Appl. Organomet. Chem. 1993, 7, 201; Sandhu, G. K.; Kaur, G.; Main Group Met. Chem. 1990, 13, 149; Tiekink, E. R. T.; J. Organomet. Chem. 1991, 408, 323. 
10. Pavia, D. L.; Lampman, G. M.; Kriz, G. S.; Introduction to Spectroscopy, Saunders College: Philadelphia, 1979.

11. Mitchel, T. N.; J. Organomet. Chem. 1973, 59, 189; Pellerito, L; Nagy, L.; Coord. Chem. Rev. 2002, 224, 111; Holeček, J.; Nádvorník, M.; Handlî́r, K.; Lyčka, A.; J. Organomet. Chem. 1983, 241, 177.

12. Howard, W. F.; Crecely, R.W.; Nelson, W. H.; Inorg. Chem. 1985, 24, 2204.

13. Davis, A.G.; Smith, P. J.; Stone, F. G. A.; Abel, E. W.; Comprehensive Organometallic Chemistry, Pergamon: New York, 1982; Jambor, R.; Dostal, L.; Ruzicka, A.; Cisarova, I.; Brus, J.; Holcapek, M.; Holecek, J.; Organometallics 2002, 21, 3996.
14. Pejchal, V.; Holecek, J.; Nadvornik, M.; Lycka, A.; Collect. Czech. Chem. Commun. 1995, 60, 1492; Wrackmeyer, B.; Application of ${ }^{119}$ Sn NMR Parameters Annu. Rep. NMR Spectrosc., 1999, 38.

15. Lockhart, T. P.; Manders, W. F.; Zukerman, J. J.; J. Am. Chem. Soc. 1985, 107, 4546; Lockhart, T. P.; Manders, W. F.; Holt, E. M.; J. Am. Chem. Soc. 1986, 108, 6611; Dalil, H.; Biesemanns, M.; Teerenstra, M.; Willem, R; Angiolini, L.; Salatelli, E.; Caretti, D.; Macromol. Chem. Phys. 2000, 201, 1266.

16. Apodaca, P.; Lee, F.; Cervantes, F.; Pannell, H. K.; Main Group Met. Chem. 2001, 24, 597; Gielen, M.; Khloufi, A.E.; Biesemans, M.; Kayser, F.; Willem, R.; Appl. Organomet. Chem. 1993, 7, 201; Brown, N. M.; PhD Thesis, Clemsom University, Clemson, SC, USA, 1972.

Received: August 6, 2007 Web Release Date: January 22, 2009 


\section{Organotin(IV) Esters of 4-Maleimido-benzoic Acid: Synthesis, Characterization and In Vitro Anti-leishmanial Effects \\ M. I. Khan, ${ }^{*, a}$ Musa Kaleem Baloch, ${ }^{b}$ Muhammad Ashfaq ${ }^{c}$ and Saima Gul ${ }^{a}$ \\ ${ }^{a}$ Department of Chemistry, Kohat University of Science \& Technology, Kohat-26000 (N-W.F.P.), Pakistan \\ ${ }^{b}$ Department of Chemistry, Gomal University, Dera Ismail Khan, Pakistan \\ 'Department of Chemistry, Islamia University, Bahawalpur, Pakistan}

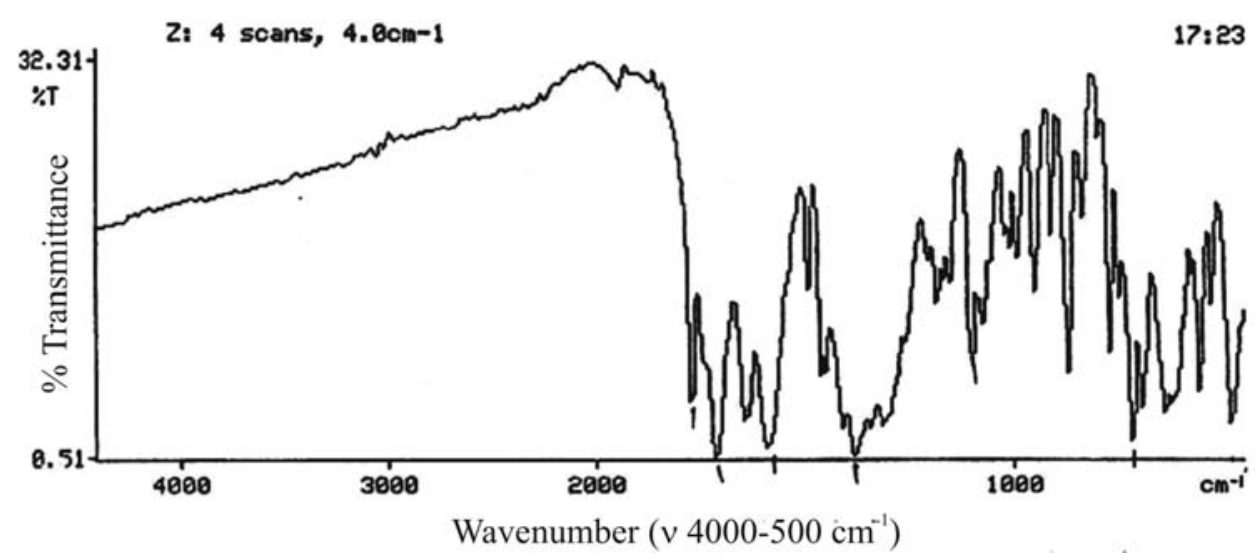

Figure S1. FT IR Spectrum of compound 1.

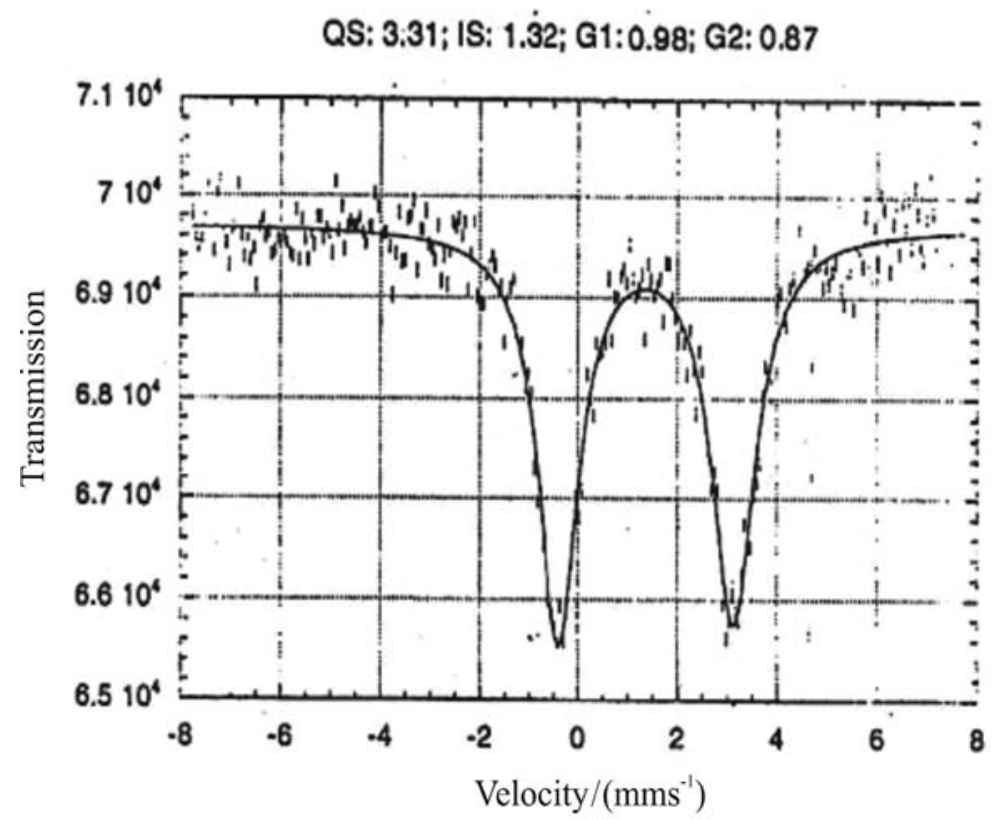

Figure S2. ${ }^{119 \mathrm{~m}}$ Sn Mössbauer Spectrum of compound $\mathbf{1}$.

*e-mail: chmikhan@hotmail.com 


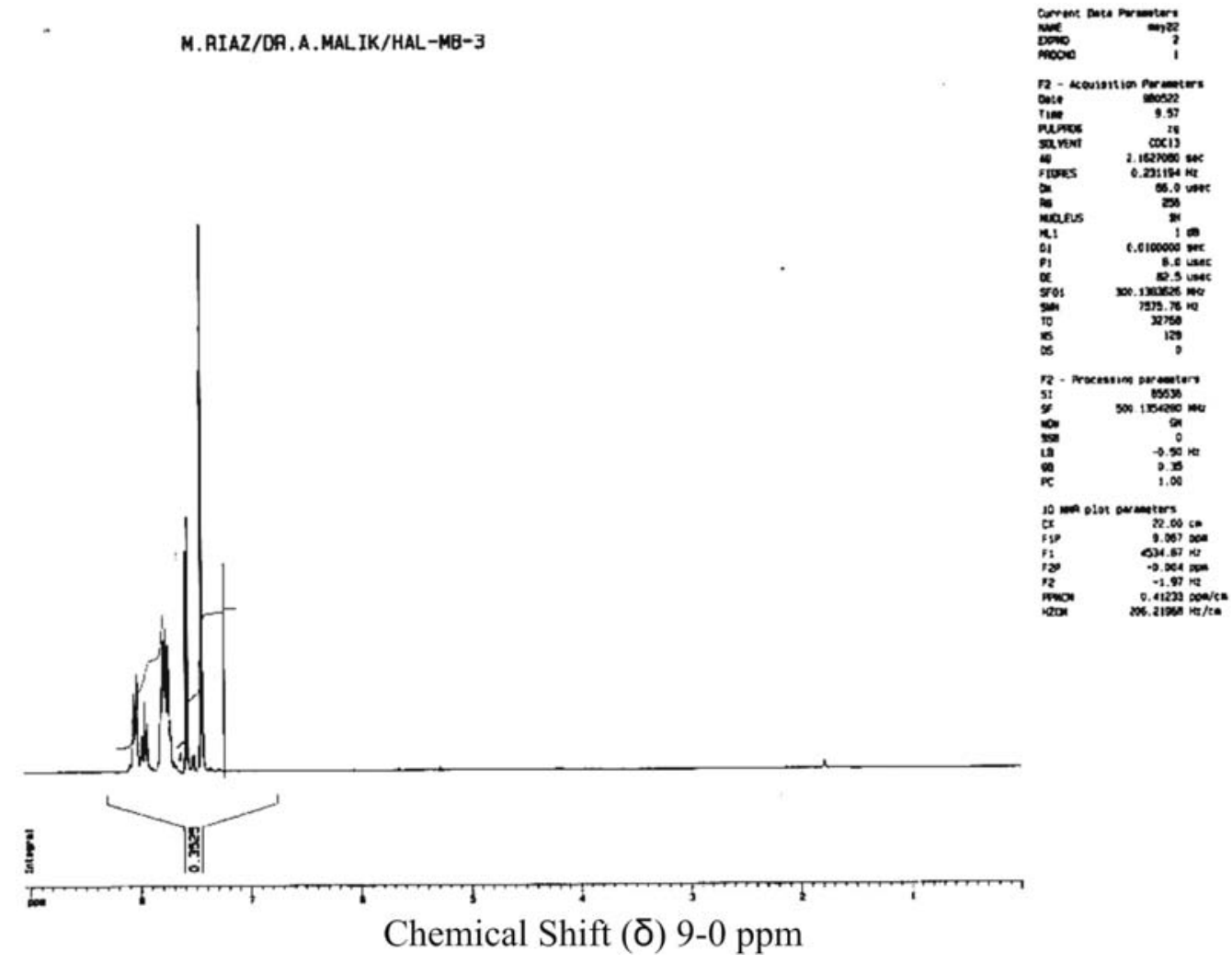

Figure S3. ${ }^{1} \mathrm{H}$ NMR Spectrum of compound $\mathbf{1}$.

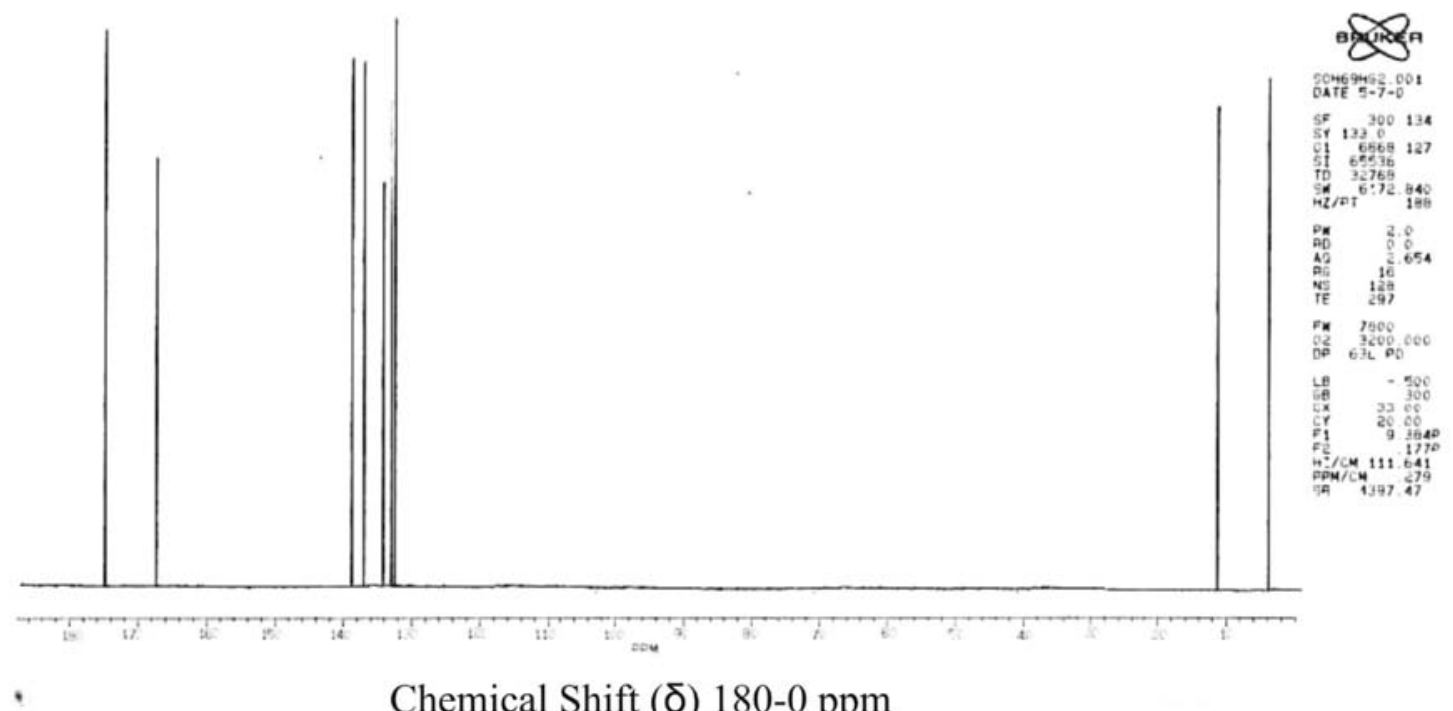

Figure S4. ${ }^{13} \mathrm{C}$ NMR Spectrum of compound 2. 


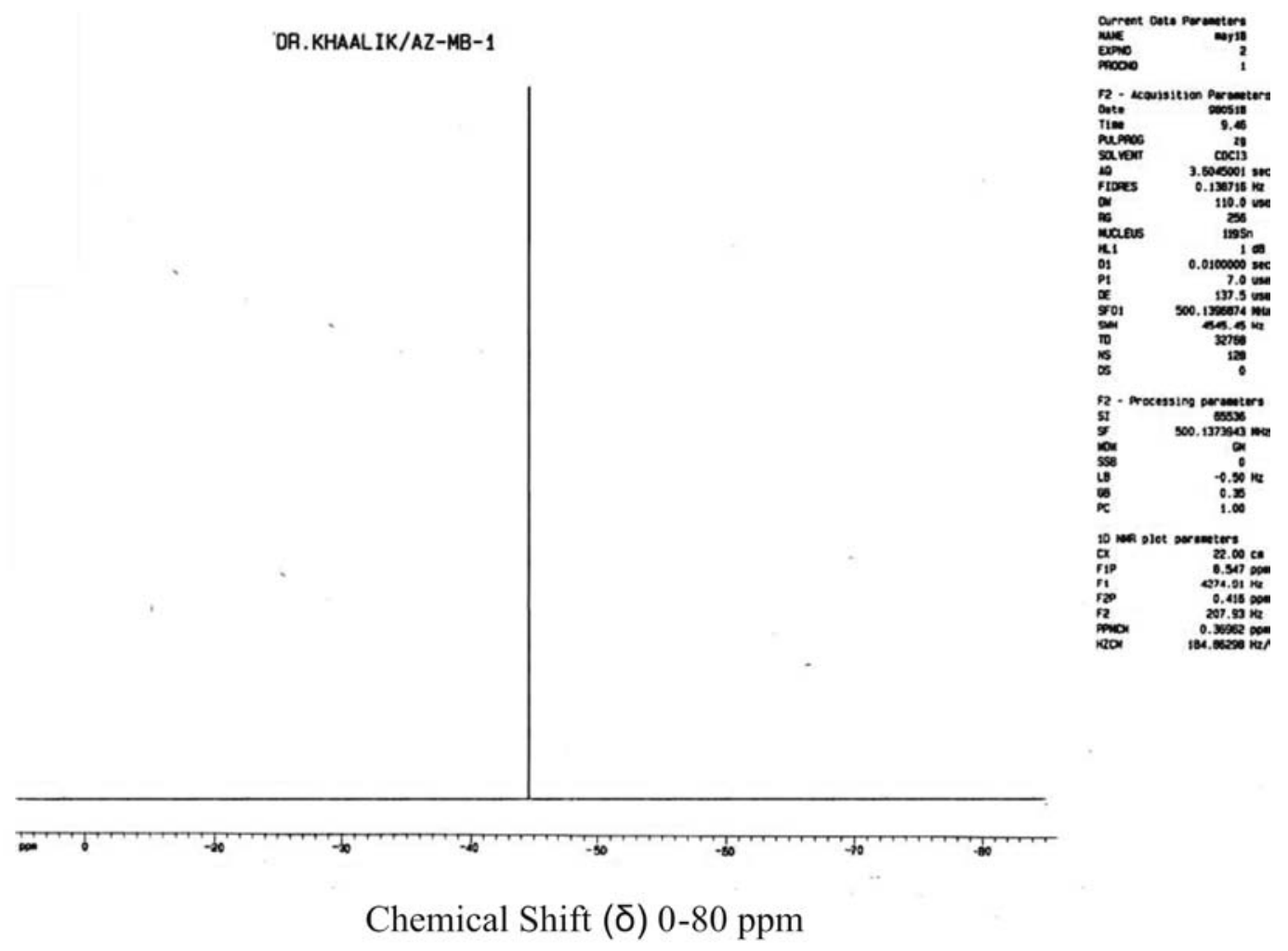

Figure S5. ${ }^{119}$ Sn NMR Spectrum of compound 6. 